\title{
Influence of Nonlinear Terms on Orbital Stability of Solitary Wave Solutions to the Generalized Symmetric Regularized-Long-Wave Equation
}

\author{
Xing-qian Ling ${ }^{1} \cdot$ Wei-guo Zhang ${ }^{2}$ (1)
}

Received: 13 January 2021 / Accepted: 31 May 2021 / Published online: 12 August 2021

(c) The Author(s) 2021

\begin{abstract}
The influence of two nonlinear terms on the orbital stability of solitary wave solutions to the generalized symmetric regularized-long-wave(gsrlw) equation is investigated in this paper. Based on the general conclusion to judge the orbit stability of solitary wave solution to the equation, the stable and unstable wave velocity intervals of solitary wave solutions to the gsrlw equation with two low order nonlinear terms are given. By appropriate transformation and scaling, the complexity caused by two high-order nonlinear terms is overcome, and the stable and unstable wave velocity intervals of solitary wave solutions to the gsrlw equation with high-order nonlinear terms are also obtained. Last, the influences of the coefficients and the order of the nonlinear terms on the stability of solitary wave solutions are studied.
\end{abstract}

Keywords Nonlinear terms · Gsrlw equation · Solitary wave solution · Orbital stability

\section{Introduction}

The symmetric regularized-long-wave equation (called srlw equation for short)

$$
u_{t t}-u_{x x}+\left(\frac{1}{2} u^{2}\right)_{x t}-u_{x x t t}=0
$$

is discovered and named by Seyler and Fenstermacher in 1984 [1], it describes a kind of weak nonlinear ion acoustic wave and space charge wave, where $\rho$ and $u$ describe the fluid velocity and electron charge density, respectively. It also appears

Wei-guo Zhang

zwgzwm@126.com

1 Business School, University of Shanghai for Science and Technology, Shanghai 200093, China

2 College of Science, University of Shanghai for Science and Technology, Shanghai 200093, China 
in many other physical phenomena and is an important model equation in physics and fluid mechanics [2-4].

Recent years, many researchers have studied the solitary wave solutions of Eq. (1) by various methods [5-8], such as $\exp (-\psi(\xi))$ extend method [5], tanh function extend method [6], analytical method [7] and exponential wave integrator pseudospectral method [8]. For the stability [9], studied the local and global well posedness problems of solutions to Eq. (1) in $H^{s}(R) \times H^{s-1}(R)$, and proved the existence and the orbit stability of elliptic periodic wave solutions with period $L$. And [10] proved the instability of periodic wave solutions to (1).

It is generally believed that the solitary wave is the result of the balance between dispersion effect and nonlinear effect. For the symmetric regularized long wave Eq. (1) with weak nonlinearity, the nonlinear term can not be ignored. When the nonlinear term is changed, the amplitude $u$ related to space and time will also change. Therefore, if the nonlinear term of symmetric regularized long wave equation is changed, whether traveling wave solutions of Eq. (1) and the stability of solutions will be affected, it is worth considering.

Gradually strengthening the nonlinear effect from Eq. (1), Shang considered the solutions of the modified regularized long wave equation in [11],

$$
\left\{\begin{array}{l}
u_{x x t}-u_{t}=\left(\rho+\frac{1}{3} u^{3}\right)_{x}, \\
\rho_{t}+u_{x}=0
\end{array}\right.
$$

[12] used the reductive perturbation method to study the amplitude modulation of symmetric regularized long-wave equation (3) with quartic nonlinearity,

$$
\left\{\begin{array}{l}
u_{x x t}-u_{t}=\left(\rho+\frac{1}{4} u^{4}\right)_{x}, \\
\rho_{t}+u_{x}=0 .
\end{array}\right.
$$

[13] used lion compact concentration theorem to consider the existence of the solutions to the generalized symmetric regularized-long-wave equation(called gsrlw equation for short) with only one high nonlinear term,

$$
\left\{\begin{array}{l}
u_{x x t}-u_{t}=\left(\rho+\frac{1}{p} u^{p}\right)_{x}, \\
\rho_{t}+u_{x}=0
\end{array}\right.
$$

and gave the convergence and error estimation of its approximate solutions. [14] designed a compact finite difference scheme to solve the gsrlw Eq. (4).

Besides, some scholars attempted to consider the gsrlw equation with two nonlinear terms, such as [15] obtained the exact solitary wave solutions and kink wave solutions to a class of gsrlw equation,

$$
\left\{\begin{array}{l}
u_{x x t}-u_{t}=\left(\rho+b_{2} u^{p+1}+b_{3} u^{2 p+1}\right)_{x}, \\
\rho_{t}+u_{x}=0
\end{array}\right.
$$

Recently, [16] studied the orbital stability of solitary wave solutions to Eq. (5) in the case of $p=1$. However, the exact stable and unstable wave velocity intervals have 
not been given in [16]. For the more general form [17], studied the orbital stability and instability of the gsrlw equation,

$$
u_{t t}-u_{x x}+f(u)_{x t}-u_{x x t t}=0,
$$

here $f \in C^{1}$, and $f(s)>0$ when $s>0$. However, [17] only gave the orbital stable or instable velocity intervals when equation (6) has only one nonlinear term $f(s)=s^{p}$ : If $f(s)=s^{p}$, when $p>5$ and $v-1 / v>\sqrt{(p-5)(p+3) / 2(p+1)}$ or $1<p<5$, the solitary wave solution to Eq. (6) is orbital stable. When $p>5$ and $v-1 / v<\sqrt{(p-5)(p+3) / 2(p+1)}$, the solution is orbital instable. So here we study the orbital stability of solitary wave solutions to the gsrlw Eq. (5) with two high order nonlinear terms, and our purpose is to obtain exact orbital stable and instable wave velocity intervals of the solitary wave solutions to the gsrlw Eq. (5) with two nonlinear terms, and find the influences of the coefficients and the order of two nonlinear terms on the stability of solitary wave solutions to Eq. (5), which should make an impact on future studies in stability of the similar equations.

In this paper, we first verify that solitary wave solution to Eq. (5) satisfy the three assumptions of Grillakis-Shatah-Strauss theory [18, 19], and give the general conclusion of orbital stability of solitary wave solutions to Eq. (5). Based on the exact expressions of the discriminant $d^{\prime \prime}(v)$ of the orbital stability of solitary wave solutions in the case of $p=2$, we obtain the specific stable and instable wave velocity intervals of the solitary wave solutions to Eq. (5). Besides, we point out the problems in [16] and correct its conclusions for the gsrlw equation. We also give the specific stable and instable wave velocity intervals for case of $p=1$. These results are new. Then by appropriate transformation and scaling, we overcome the complexity caused by the two high-order nonlinear terms, and obtain the stable and instable wave velocity ranges of solitary wave solutions to Eq. (5) in the case of $p>2$. Last, we analysis the influence of the coefficients and the order of nonlinear terms on the orbital stability and instability of solutions to the gsrlw Eq. (5), and get the conclusion that the ratio $\frac{b_{2}}{\sqrt{b_{3}}}$ of the coefficients $b_{2}, b_{3}$ affects the orbital stable and instable wave velocity intervals, and the influence of the order of nonlinear terms on the orbital stability of solutions may be stronger than the influence of the coefficient of nonlinear terms.

For the convenient in the following discussions, here quote two exact solitary wave solutions of Eq. (5) in [15] as follows.

Lemma 1 Assuming $v^{2}-1>0, v$ is velocity.

(1) If $b_{3} v>0$, or $b_{3}=0$ and $b_{2} v>0$, then equation (5) has a bell-shaped solitary wave solution

$$
\left\{\begin{array}{l}
u_{1}(x-v t)=\varphi_{1}(\xi)=\varphi_{1}(x-v t), \\
\rho_{1}(x-v t)=\psi_{1}(\xi)=\frac{1}{v} \varphi_{1}(x-v t)
\end{array}\right.
$$

among which 


$$
\varphi_{1}(\xi)=\left(\frac{A_{1} \operatorname{sech}^{2} \frac{\alpha_{1}}{2}\left(\xi+\xi_{0}\right)}{2+B_{1} \operatorname{sech}^{2} \frac{\alpha_{1}}{2}\left(\xi+\xi_{0}\right)}\right)^{\frac{1}{p}},
$$

where

$$
\begin{aligned}
& A_{1}=\frac{(p+2) \sqrt{p+1}\left(v^{2}-1\right)}{\sqrt{v\left[(p+1) b_{2}^{2} v-b_{3}\left(1-v^{2}\right)(p+2)^{2}\right]}}, \quad B_{1}=-1+\frac{b_{2} v}{(p+2)\left(v^{2}-1\right)} A_{1}, \\
& \alpha_{1}=\frac{p}{v} \sqrt{v^{2}-1} .
\end{aligned}
$$

(2) If $p$ makes $(e)^{\frac{1}{p}}$ meaningful, where e is negative, when $b_{3} v>0$, or $b_{3}=0$ and $b_{2} v>0$, equation (5) has the following solitary wave solutions,

$$
\left\{\begin{array}{l}
u_{2}(x-v t)=\varphi_{2}(\xi)=\varphi_{2}(x-v t), \\
\rho_{2}(x-v t)=\frac{1}{v} \varphi_{2}(x-v t),
\end{array}\right.
$$

among which

$$
\varphi_{2}(\xi)=\left(\frac{A_{2} \operatorname{sech}^{2} \frac{\alpha_{2}}{2}\left(\xi+\xi_{0}\right)}{2+B_{2} \operatorname{sech}^{2} \frac{\alpha_{2}}{2}\left(\xi+\xi_{0}\right)}\right)^{\frac{1}{p}},
$$

where

$$
A_{2}=-A_{1}, \quad B_{2}=-B_{1}-2, \quad \alpha_{2}=\alpha_{1} .
$$

\section{General Conclusions of the Discriminant $d^{\prime \prime}(v)$ of the Orbital Stability of the Solitary Wave Solution to the gsrlw Equation}

Here introduce some background knowledge before giving the three assumptions of Grillakis-Shatah-Strauss theory [18, 19]. The gsrlw Eq. (5) can be written into the following Hamilton system,

$$
\frac{d \vec{u}}{d t}=J E^{\prime}(\vec{u}), \quad \vec{u} \in X
$$

where

$$
\begin{aligned}
& E(\vec{u})=-\int_{-\infty}^{+\infty}\left(\rho u+\frac{b_{2}}{p+2} u^{p+2}+\frac{b_{3}}{2 p+2} u^{2 p+2}\right) d x \\
& E^{\prime}(\vec{u})=\left[\begin{array}{c}
-\rho-b_{2} u^{p+1}-b_{3} u^{2 p+1} \\
-u
\end{array}\right], \quad J=\frac{\partial}{\partial x}\left[\begin{array}{cc}
\left(1-\frac{\partial^{2}}{\partial x^{2}}\right)^{-1} & 0 \\
0 & 1
\end{array}\right],
\end{aligned}
$$


here $E$ is one natural invariant of Eq. (5), and $E^{\prime}(\vec{u})$ is the Frechet derivative of $E$, $J$ is a skew symmetric operator, and $X=H^{1}(R) \times L^{2}(R)$ is a Hilbert space with the inner product

$$
\left(\vec{u}_{1}, \vec{u}_{2}\right)=\int\left(u_{1} u_{2}+\rho_{1} \rho_{2}+u_{1 x} u_{2 x}\right) d x, \quad \forall \vec{u}_{1}, \vec{u}_{2} \in X .
$$

There exists a natural isomorphism $I: X \rightarrow X^{*}$ with its dual space $X^{*}$, satisfying

$$
\langle I u, v\rangle=(u, v), \quad I=\left[\begin{array}{cc}
1 & 0 \\
0 & 1-\Delta
\end{array}\right] .
$$

Let $T$ be a unitary operator group with single parameter, which is defined as,

$$
T(s) u(\cdot)=u(\cdot-s), \quad \forall s \in R, \vec{u}(\cdot) \in X .
$$

Apparently, here $\quad T^{\prime}(0)=\left[\begin{array}{cc}-\partial / \partial x & 0 \\ 0 & -\partial / \partial x\end{array}\right]$. Define $\quad T^{\prime}(0)=J B \quad$ and $V(\vec{u})=-\frac{1}{2}\langle B \vec{u}, \vec{u}\rangle$, then we can get

$$
B=\left[\begin{array}{cc}
\Delta-1 & 0 \\
0 & -1
\end{array}\right], \quad V(\vec{u})=-\frac{1}{2}\langle B \vec{u}, \vec{u}\rangle=\frac{1}{2} \int\left(u^{2}-u_{x x} u+\rho^{2}\right) d x,
$$

From (16), we know that solitary wave solutions to Eq. (5) can be expressed as,

$$
\phi=T(v t) \vec{\phi}_{i}(x)(i=1,2),
$$

where $\vec{\phi}_{1}(x)=\left(\begin{array}{c}u_{1}(x) \\ \rho_{1}(x)\end{array}\right), \vec{\phi}_{2}(x)=\left(\begin{array}{c}u_{2}(x) \\ \rho_{2}(x)\end{array}\right)$ are given by (7), (10), respectively.

To prevent repetition, we denote $\phi_{v}(x)$ as one of $u_{1}(x)$ and $u_{2}(x)$.

Next, we will show that $T(v t) \vec{\phi}_{v}(x)$ satisfies three assumptions of Grillakis-Shatah-Strauss theory. Here we introduce the assumption in [18, 19] as the following lemma.

Lemma 2 (Existence of Solutions) Set $X=H^{1}(R) \times L^{2}(R)$, for each $x_{0} \in X$, there exits $t_{0}>0$ depending only on $\mu$, where $\left\|u_{0}\right\| \leq \mu$, and there exists a solution $u$ of (13) in the interval $\left[0, t_{0}\right)$ such that $(a) u(0)=u_{0} ;(b) E(u(t))=E\left(u_{0}\right), Q(u(t))=Q\left(u_{0}\right)$ for $t \in\left[0, t_{0}\right)$.

By semigroup theory [20], the existence of the initial value problem of equation can be proved by the similar method in [17], so we omit the proof process here. Next, we give the Lemma 3, that is the assumption 2 in $[18,19]$.

Lemma 3 There exist real numbers $v_{1}, v_{2}$ and a mapping $v \rightarrow \phi_{v}$ from the open interval $\left(v_{1}, v_{2}\right)$ into $X$ which is $C^{1}$ such that for each $v \in\left(v_{1}, v_{2}\right), E^{\prime}\left(\vec{\varphi}_{v}\right)+v V^{\prime}\left(\vec{\varphi}_{v}\right)=0$.

Proof Take $\vec{\phi}_{v}=\left[\begin{array}{l}\varphi_{v} \\ \psi_{v}\end{array}\right]$ into Eq. (5), we can get 


$$
\left\{\begin{array}{l}
\varphi_{v \xi}=v \psi_{v \xi} \\
-v \varphi_{v \xi \xi \xi}+v \varphi_{v \xi}=\left(\psi_{v}+b_{2} \varphi_{v}^{p+1}+b_{3} \varphi_{v}^{2 p+1}\right)_{\xi}
\end{array}\right.
$$

We make integration on the both sides of Eq. (18), and set the constant as 0, then we have

$$
\left\{\begin{array}{l}
\varphi_{v}=v \psi_{v} \\
-v \varphi_{v \xi \xi}+v \varphi_{v \xi}=\psi_{v}+b_{2} \varphi_{v}^{p+1}+b_{3} \varphi_{v}^{2 p+1}
\end{array}\right.
$$

Take $\vec{\phi}_{v}=\left[\begin{array}{l}\varphi_{v} \\ \psi_{v}\end{array}\right]$ into $E^{\prime}\left(\vec{\phi}_{v}\right)+v V^{\prime}\left(\vec{\phi}_{v}\right)$, we can get

$$
\begin{aligned}
E^{\prime}\left(\vec{\phi}_{v}\right)+v V^{\prime}\left(\vec{\phi}_{v}\right) & =\left[\begin{array}{c}
-\psi_{v}-b_{2} \varphi_{v}^{p+1}-b_{3} \varphi_{v}^{2 p+1} \\
-\varphi_{v}
\end{array}\right]+v\left[\begin{array}{c}
\varphi_{v}-\varphi_{v \xi \xi} \\
\psi_{v}
\end{array}\right] \\
& =\left[\begin{array}{c}
-\psi_{v}-b_{2} \varphi_{v}^{p+1}-b_{3} \varphi_{v}^{2 p+1}+v \varphi_{v}-v \varphi_{v \xi \xi} \\
-\varphi_{v}+v \psi_{v}
\end{array}\right] .
\end{aligned}
$$

So $E^{\prime}\left(\vec{\phi}_{v}\right)+v V^{\prime}\left(\vec{\phi}_{v}\right)=0$ is established.

Then we give the assumption 3 in $[18,19]$ as Lemma 4 here.

Lemma 4 For each $\omega \in\left(\omega_{1}, \omega_{2}\right), H_{v}$ has exactly one negative simple eigenvalue and has its kernel spanned by $T^{\prime}(0) \phi_{\omega}$, and the rest of its spectrum is positive and bounded away from zero.

Proof Considering the operator $H_{v}=E^{\prime \prime}\left(\vec{\varphi}_{v}\right)+v V^{\prime \prime}\left(\vec{\varphi}_{v}\right)$, and making spectrum analysis, where

$$
E^{\prime \prime}(\vec{u})=\left[\begin{array}{cc}
-b_{2}(p+1) u^{p}-b_{3}(2 p+1) u^{2 p} & -1 \\
-1 & 0
\end{array}\right], \quad V^{\prime \prime}(\vec{u})=\left[\begin{array}{cc}
1-\Delta & 0 \\
0 & 1
\end{array}\right] .
$$

$\forall \quad \vec{u}_{1}, \vec{u}_{2} \in H^{1}(R) \times L^{2}(R)$, we have $\left\langle H_{v} \vec{u}_{1}, \vec{u}_{2}\right\rangle=\left\langle H_{v} \vec{u}_{2}, \vec{u}_{1}\right\rangle$, which means $H_{v}$ is a self-adjoint operator, and $I^{-1} H_{v}$ is a bounded self-adjoint operator on $X$. And eigenvalues $\lambda$ of $H_{v}$ make $H_{v}-\lambda I$ irreversible. Then from (19), we can get

$$
L \varphi_{v x}=0,
$$

where $L=-b_{2}(p+1) \varphi_{v}^{p}-b_{3}(2 p+1) \varphi_{v}^{2 p}-v \frac{\partial^{2}}{\partial x^{2}}+v-\frac{1}{v}$. Since $v-\frac{1}{v}>0$, when $|\xi| \rightarrow \infty,-b_{2}(p+1) \varphi_{v}^{p}-b_{3}(2 p+1) \varphi_{v}^{2 p} \rightarrow 0$, from Weyl essential spectrum theory, we have $\sigma_{\text {ess }}(L)=\left[v-\frac{1}{v},+\infty\right)$. From (23), we can know $x=0$ is the only zero point of $\varphi_{v x}$, then according to Sturm-Liouville theory, we can get that 0 is the second eigenvalue of $L$. So under the condition $v>1, L$ only has one negative eigenvalue $-\sigma^{2}$, and its corresponding eigenfunction is $\chi$, that is $L \chi=-\sigma^{2} \chi$.

Now definite $d(v): R \rightarrow R$ as $d(v)=E\left(\vec{\phi}_{v}\right)+v V\left(\vec{\phi}_{v}\right)$, we have 


$$
d^{\prime}(v)=\left\langle E^{\prime}\left(\vec{\phi}_{v}\right), \frac{\partial \vec{\phi}_{v}}{\partial v}\right\rangle+v\left\langle V^{\prime}\left(\vec{\phi}_{v}\right), \frac{\partial \vec{\phi}_{v}}{\partial v}\right\rangle+V\left(\vec{\phi}_{v}\right)=V\left(\vec{\phi}_{v}\right) .
$$

According to the orbital stability theory proposed by Grillakis-Shatah-Strauss and Lemmas 2-4, we can get the following general conclusion of orbital stability of solitary wave solution to the gsrlw Eq. (5).

Theorem 1 Suppose $b_{2} b_{3} \neq 0, b_{3} \geq 0, \quad v>1$, and $\vec{\phi}_{v}=T(v t) \vec{\phi}(x)$ is the solution of equation (5). (1) If $d^{\prime \prime}(v)>0, \vec{\phi}_{v}$ is orbital stable; (2) If $d^{\prime \prime}(v)<0, \vec{\phi}_{v}$ is orbital instable.

Remark 1 Since the skew-symmetric operator $J$ is not onto, by making similarly deduction in [16], we can obtain the conclusion that if $d^{\prime \prime}(v)<0, \vec{\phi}_{v}(x)$ is orbital instable in Theorem 1.

From Theorem 1, in order to consider the orbital stability of solitary wave solutions to Eq. (5), we only need to consider the sigh of the second derivative $d^{\prime \prime}(v)$ of $d(v)=E\left(\vec{\phi}_{v}\right)+v V\left(\vec{\phi}_{v}\right)$, here

$$
d^{\prime}(v)=\frac{1}{2} \int_{R}\left(u^{2}-u_{x x} u+\rho^{2}\right) d x=\frac{1}{2} \int_{R}\left(\left(1+\frac{1}{v^{2}}\right) u^{2}+u_{x}^{2}\right) .
$$

Then we consider the orbital stability of solitary wave solutions to the gsrlw equation in the case of $p=1, p=2, p>2$. For the convenient of discussion, here always assume $b_{3}>0$.

\section{Orbital Stability of Solitary Wave Solution to Eq. (5) When $p=1,2$}

Here, we consider the case of $p=2$ first. Solitary wave solution (7) can be rewritten into

$$
u_{1}(\xi)=\left(\frac{A_{1} \operatorname{sech}^{2} \frac{\alpha}{2}\left(\xi+\xi_{0}\right)}{2+B_{1} \operatorname{sech}^{2} \frac{\alpha}{2}\left(\xi+\xi_{0}\right)}\right)^{1 / 2}, \quad(\xi=x-v t),
$$

where $A_{1}=\frac{4 \sqrt{3}\left(v^{2}-1\right)}{\sqrt{3 b_{2}^{2} v^{2}+16 b_{3}\left(v^{2}-1\right)}}, B_{1}=\frac{\sqrt{3} b_{2} v}{\sqrt{3 b_{2}^{2} v^{2}+16 b_{3}\left(v^{2}-1\right)}}-1, \alpha=2 \sqrt{1-\frac{1}{v^{2}}}$. Substituting (25) into (24), we have

$$
\begin{aligned}
& d^{\prime}(v)=\frac{1}{2} \int_{R}\left(\left(1+\frac{1}{v^{2}}\right) u_{1}^{2}+u_{1 \xi}^{2}\right) d \xi \\
& =\frac{1}{2} \int_{R}\left(\frac{2 A_{1} e^{\alpha\left(\xi+\xi_{0}\right)}}{2 B_{1} e^{\alpha\left(\xi+\xi_{0}\right)}+\left(e^{\alpha\left(\xi+\xi_{0}\right)}+1\right)^{2}}\right)\left(1+\frac{1}{v^{2}}+\frac{\alpha^{2}\left(e^{2 \alpha\left(\xi+\xi_{0}\right)}-1\right)^{2}}{\left(2\left(e^{\alpha\left(\xi+\xi_{0}\right)}+1\right)^{2}+4 B_{1} e^{\alpha\left(\xi+\xi_{0}\right)}\right)^{2}}\right) d \xi
\end{aligned}
$$

let $z=e^{\alpha\left(\xi+\xi_{0}\right)}$, then 


$$
\begin{aligned}
d^{\prime}(v)= & \int_{0}^{+\infty} \frac{1}{\alpha z}\left(\frac{A_{1} z}{2 B_{1} z+(z+1)^{2}}\right)\left(1+\frac{1}{v^{2}}+\frac{\alpha^{2}\left(z^{2}-1\right)^{2}}{\left(2(z+1)^{2}+4 B_{1} z\right)^{2}}\right) d z \\
= & \frac{A_{1}}{2 \alpha \sqrt{-B_{1}\left(B_{1}+2\right)}}\left(1+\frac{1}{v^{2}}+\frac{\alpha^{2}}{8 B_{1}\left(B_{1}+2\right)}\right) \\
& \left(\pi-2 \arctan \left(\frac{B_{1}+1}{\sqrt{-B_{1}\left(B_{1}+2\right)}}\right)\right)+\frac{\alpha A_{1}\left(B_{1}+1\right)}{8 B_{1}\left(B_{1}+2\right)}
\end{aligned}
$$

and substitute $A_{1}=\frac{4 \sqrt{3}(v-1 / v)}{\sqrt{3 b_{2}^{2}+16 b_{3}(v-1 / v)}}, B_{1}=-1+\frac{\sqrt{3} b_{2}}{\sqrt{3 b_{2}^{2}+16 b_{3}(v-1 / v)}}$ into (27), we can get

$$
d^{\prime}(v)=\frac{\sqrt{3}}{8 \sqrt{b_{3} v}}\left(\frac{v^{2}+3}{v}-k^{2}\right)\left(\pi-2 \arctan \frac{\sqrt{3} b_{2}}{4 \sqrt{b_{3}(v-1 / v)}}\right)-\frac{3 b_{2} \sqrt{v^{2}-1}}{16 b_{3} v} .
$$

Similarly, for the solitary wave solution (10), we have

$$
\bar{d}^{\prime}(v)=-\frac{\sqrt{3}}{8 \sqrt{b_{3} v}}\left(\frac{v^{2}+3}{v}-k^{2}\right)\left(\pi+2 \arctan \frac{\sqrt{3} b_{2}}{4 \sqrt{b_{3}(v-1 / v)}}\right)-\frac{3 b_{2} \sqrt{v^{2}-1}}{16 b_{3} v} .
$$

By further deriving $d^{\prime}(v)$ and $\bar{d}^{\prime}(v)$, we have

$$
\begin{aligned}
d^{\prime \prime}(v)= & \frac{k^{2}}{2 b_{2} v^{3} \sqrt{v^{2}-1}\left(m-k^{2}\right)}\left(\left(v^{2}-1\right)\left(v^{2}-1-v k^{2}\right)+4\left(v^{2}+1\right)\right) \\
& -\frac{k \sqrt{v^{2}-1}}{4 m b_{2} v^{2}}\left(k^{2}+v-\frac{9}{v}\right) D_{1}, \\
\bar{d}^{\prime \prime}(v)= & \frac{k^{2}}{2 b_{2} v^{3} \sqrt{v^{2}-1}\left(m-k^{2}\right)}\left(\left(v^{2}-1\right)\left(v^{2}-1-v k^{2}\right)+4\left(v^{2}+1\right)\right) \\
& +\frac{k \sqrt{v^{2}-1}}{4 m b_{2} v^{2}}\left(k^{2}+v-\frac{9}{v}\right) D_{2},
\end{aligned}
$$

where $m=v-\frac{1}{v}, k^{2}=\frac{3 b_{2}^{2}}{16 b_{3}}, D_{1}=\pi-2 \arctan \frac{k}{m}, D_{2}=\pi+2 \arctan \frac{k}{m}$.

It's easy to verify that when $b_{2}>0, \frac{k}{m} D_{1} \in(0,2), \frac{k}{m} D_{2} \in(0,+\infty)$; when $b_{2}<0$, $\frac{k}{m} D_{1} \in(-\infty, 0), \frac{k}{m} D_{2} \in(-2,0)$. In order to consider the orbital stability of the solitary wave solution (25), we need to consider the sign of $d^{\prime \prime}(v)$. Here we discuss the orbital stability of (25) first.

(1) When $1<v<3$.

Since $\frac{k}{m} D_{1} \in(0,2)$ when $b_{2}>0$, if $\frac{9}{v}-v>k^{2}$ is established, $-\frac{k \sqrt{v^{2}-1}}{4 b_{2} v^{2} m}\left(k^{2}+v-\frac{9}{v}\right) D_{1}>0$. If and only if $\frac{\left(v^{2}-1\right)\left(v^{2}-1-v k^{2}\right)+4\left(v^{2}+1\right)}{m-k^{2}}>0$, namely $\left(v^{2}-1\right) v+\frac{4\left(v^{2}+1\right) v}{v^{2}-1-v k^{2}}>0$, we have $d^{\prime \prime}(v)>0$. After calculation, we can get $k^{2}>\frac{\left(v^{2}-1\right)^{2}+4 v^{2}+4}{v\left(v^{2}-1\right)}$. Since $\frac{\left(v^{2}-1\right)^{2}+4 v^{2}+4}{v\left(v^{2}-1\right)}>\frac{9}{v}-v$, we can't obtain the interval which makes $d^{\prime \prime}(v)>0$ in this case. 
If $\frac{9}{v}-v<k^{2}$, then $-\frac{k \sqrt{v^{2}-1}}{4 b_{2} v^{2} m}\left(k^{2}+v-\frac{9}{v}\right) D_{1}<0$. Since $\frac{k}{m} D_{1} \in(0,2)$, in order to consider the orbital stability of $u_{1}(\xi)$, we take $\frac{k}{m} D_{1}=2$, and consider when $d^{\prime \prime}(v)>0$. After simplification, we have

$$
\begin{aligned}
d^{\prime \prime}(v)= & \frac{k^{2}}{2 b_{2} v^{3} \sqrt{v^{2}-1}\left(m-k^{2}\right)}\left(\left(v^{2}-1\right)\left(v^{2}-1-v k^{2}\right)+4\left(v^{2}+1\right)\right. \\
& \left.-\frac{v^{2}-1}{2} \frac{v\left(m-k^{2}\right)}{k^{2}}\left(k^{2}+v-\frac{9}{v}\right) D_{1}\right) \\
= & \frac{k^{2}}{2 b_{2} v^{3} \sqrt{v^{2}-1}\left(m-k^{2}\right)}\left(4\left(v^{2}+1\right)-\frac{1}{k^{2}}\left(v^{2}-1\right)\left(v^{2}-1-v k^{2}\right)\left(v-\frac{9}{v}\right)\right) \\
= & \frac{1}{2 b_{2} v^{3} \sqrt{v^{2}-1}\left(m-k^{2}\right)}\left(k^{2}\left(4 v^{2}+4+v^{4}-10 v^{2}+9\right)-\left(v^{2}-1\right)^{2}\left(v-\frac{9}{v}\right)\right) \\
= & \frac{1}{2 b_{2} v^{3} \sqrt{v^{2}-1}} \frac{k^{2}\left(v^{4}-6 v^{2}+13\right)-\left(v^{2}-1\right)^{2}\left(v-\frac{9}{v}\right)}{m-k^{2}} .
\end{aligned}
$$

Since $v-\frac{9}{v}<0$ when $1<v<3$, in order to make $d^{\prime \prime}(v)>0$, we have $m-k^{2}>0$, namely $v-\frac{1}{v}>k^{2}$ from (30). So we can obtain $\frac{9}{v}-v<k^{2}<v-\frac{1}{v}$ when $\sqrt{5}<v<3$.

(2) When $v>3$

Now $v-\frac{9}{v}>0$ is always established, so $\frac{9}{v}-v<0$. Similar to the discussion in (1), we take $\frac{k^{v}}{m} D_{1}=2$ and consider when $d^{\prime \prime}(v)>0$. From (30), we can get

$$
\frac{k^{2}\left(v^{4}-6 v^{2}+13\right)-\left(v^{2}-1\right)^{2}\left(v-\frac{9}{v}\right)}{v-\frac{1}{v}-k^{2}}>0 .
$$

Since $\frac{\left(v^{2}-1\right)^{2}\left(v-\frac{9}{v}\right)}{v^{4}-6 v^{2}+13}<v-\frac{9}{v}<v-\frac{1}{v}$, we have when $\frac{\left(v^{2}-1\right)^{2}\left(v-\frac{9}{v}\right)}{v^{4}-6 v^{2}+13}<k^{2}<v-\frac{1}{v}$, $d^{\prime \prime}(v)>0$.

Then we consider the instability of the solitary wave solution $u_{1}(\xi)$.

(1) When $1<v<3$

If $b_{2}>0$, we have $-\frac{k \sqrt{v^{2}-1}}{4 b_{2} v^{2} m}\left(k^{2}+v-\frac{9}{v}\right) D_{1}<0$ when $\frac{9}{v}-v<k^{2}$. In order to make $d^{\prime \prime}(v)<0$, we only need to consider when $\frac{\left(v^{2}-1\right)\left(v^{2}-1-v k^{2}\right)+4\left(v^{2}+1\right)}{m-k^{2}}<0$ is established. After simplification, we can get that when $\max \left\{v-\frac{1}{v}, \frac{9}{v}-v\right\}<k^{2}<\frac{\left(v^{2}-1\right)^{2}+4 v^{2}+4}{v\left(v^{2}-1\right)}, d^{\prime \prime}(v)<0$.

If $b_{2}<0$, we have $\frac{k}{m} D_{1} \in(-\infty, 0)$, and $-\frac{k \sqrt{v^{2}-1}}{4 b_{2} v^{2} m}\left(k^{2}+v-\frac{9}{v}\right) D_{1}<0$. Then only if when $\frac{\left(v^{2}-1\right)\left(v^{2}-1-v k^{2}\right)+4\left(v^{2}+1\right)}{m-k^{2}}>0$, we have $d^{\prime \prime}(v)<0$. After calculation, we can obtain that when $k^{2}>\frac{\left(v^{2}-1\right)^{2}+4 v^{2}+4}{v\left(v^{2}-1\right)}$ or $k^{2}<v-\frac{1}{v}, d^{\prime \prime}(v)<0$.

(2) When $v>3$, since $\frac{9}{v}-v<k^{2},-\frac{k \sqrt{v^{2}-1}}{4 b_{2} v^{2} m}\left(k^{2}+v-\frac{9}{v}\right) D_{1}<0$ is always established. Similarly, we can get the following conclusions: If $b_{2}>0$, when $v-\frac{1}{v}<k^{2}<\frac{\left(v^{2}-1\right)^{2}+4 v^{2}+4}{v\left(v^{2}-1\right)}$, $d^{\prime \prime}(v)<0$; if $b_{2}<0$, when $k^{2}>\frac{\left(v^{2}-1\right)^{2}+4 v^{2}+4}{v\left(v^{2}-1\right)}$ or $k^{2}<v-\frac{1}{v}, d^{\prime \prime}(v)<0$. 
In conclusion, we can get the following theorem.

Theorem 2 Suppose $p=2, b_{3}>0, k=\frac{\sqrt{3} b_{2}}{4 \sqrt{b_{3}}}$.

(1) If $b_{2}>0$, when $\sqrt{5}<v<3$ and $\frac{9}{v}-v<k^{2}<v-\frac{1}{v}$, or when $v>3$ and $\frac{\left(v^{2}-1\right)^{2}\left(v-\frac{9}{v}\right)}{v^{4}-6 v^{2}+13}<k^{2}<v-\frac{1}{v}$, the solitary wave solution $u_{1}(\xi)$ is orbital stable.

(2) If $b_{2}>0$, when $1<v<3$ and $\max \left\{v-\frac{1}{v}, \frac{9}{v}-v\right\}<k^{2}<\frac{\left(v^{2}-1\right)^{2}+4 v^{2}+4}{v\left(v^{2}-1\right)}$ or when $v>3$ and $v-\frac{1}{v}<k^{2}<\frac{\left(v^{2}-1\right)^{2}+4 v^{2}+4}{v\left(v^{2}-1\right)}$, the solitary wave solution $u_{1}(\xi)$ is orbital instable.

(3) If $b_{2}<0$, when $k^{2}>\frac{\left(v^{2}-1\right)^{2}+4 v^{2}+4}{v\left(v^{2}-1\right)}$ or $k^{2}<v-\frac{1}{v}$, the solitary wave solution $u_{1}(\xi)$ is orbital instable.

For the solitary wave solution $u_{2}(\xi)$, we can obtain the following conclusions similarly.

Theorem 3 Suppose $p=2, b_{3}>0, k=\frac{\sqrt{3} b_{2}}{4 \sqrt{b_{3}}}$.

(1) If $b_{2}<0$, when $\sqrt{5}<v<3$ and $\frac{9}{v}-v<k^{2}<v-\frac{1}{v}$, or when $v>3$ and $\frac{\left(v^{2}-1\right)^{2}\left(v-\frac{9}{v}\right)}{v^{4}-6 v^{2}+13}<k^{2}<v-\frac{1}{v}$, the solitary wave solution $u_{2}(\xi)$ is orbital instable.

(2) If $b_{2}<0$, when $1<v<3$ and $\max \left\{v-\frac{1}{v}, \frac{9}{v}-v\right\}<k^{2}<\frac{\left(v^{2}-1\right)^{2}+4 v^{2}+4}{v\left(v^{2}-1\right)}$ or when $v>3$ and $v-\frac{1}{v}<k^{2}<\frac{\left(v^{2}-1\right)^{2}+4 v^{2}+4}{v\left(v^{2}-1\right)}$, the solitary wave solution $u_{2}(\xi)$ is orbital stable.

(3) If $b_{2}>0$, when $k^{2}>\frac{\left(v^{2}-1\right)^{2}+4 v^{2}+4}{v\left(v^{2}-1\right)}$ or $k^{2}<v-\frac{1}{v}$, the solitary wave solution $u_{2}(\xi)$ is orbital stable.

Remark 2 Since the case of $p=1$ has been discussed in [16], we omit the computing process here. And for the convenience to comprehend, we unify the velocity $c$ in [16] as $v$ here. And here introduce the discriminant $d^{\prime \prime}(v)$ in the case of $p=1$,

$$
\begin{aligned}
d^{\prime \prime}(v)= & \frac{\sqrt{v^{2}}}{12 b_{3} v^{2}}\left(-3 D_{1} k \sqrt{\frac{v}{v^{2}-1}}\left(3 v-\frac{3}{v}-k^{2}\right)\right. \\
& \left.+\frac{2\left(16 v^{2}+7 k^{2} v^{3}-\left(8+3 k^{4}\right) v^{2}-7 k^{2} v+16\right.}{v\left(v^{2}-1+k^{2} v\right)}\right) .
\end{aligned}
$$

However, it can be proved that equation (61) in [16]

$$
7 v^{4}+k^{2} c^{3}+10 v^{2}-k^{2} v+7=7(v-1)^{2}+k^{2} v^{2}(v-1)+24 v^{2}>0
$$


is always established, then equation (64) in [16]

$$
7 v^{4}+k^{2} v^{3}+10 v^{2}-k^{2} v+7<0
$$

is always incorrect. Similarly, in the case of $3 v-\frac{3}{v}-k^{2}>0$, it can be proved that equation (62)

$$
\begin{aligned}
& 16 v^{4}+7 k^{2} v^{3}-\left(8+3 k^{4}\right) v^{2}-7 k^{2} v+16 \\
& =7\left(v^{2}-1\right)^{2}+k^{2} v\left(v^{2}-1\right)+24 v^{2}+3\left(3\left(v^{2}-1\right)-k^{2} v\right)\left(v^{2}-1+k^{2} v\right)>0
\end{aligned}
$$

is always established, and equation (63) is always incorrect. And in the case of $3 v-\frac{3}{v}-k^{2}<0$

$$
0<v-\frac{1}{v}<\frac{-7 k^{4}+\sqrt{241 k^{2}-1536}}{32}
$$

can be obtained by solving (63) in [16]. According to the above analysis, we make improvements and corrections here for the theorem 9, 10 in [16], and obtain the following theorems.

Theorem 4 Suppose $p=1, b_{3}>0, k=\frac{\sqrt{2} b_{2}}{3 b_{3}}$.

(1) When $v-\frac{1}{v} \geq \frac{k^{2}}{3}$, whether $b_{2}>0$ or $b_{2}<0$, the solitary wave solution $u_{1}(\xi)$ is always orbital stable.

(2) When $v-\frac{1}{v}<\frac{k^{2}}{3}$, if $b_{2}>0$, when $\frac{-7 k^{4}+\sqrt{241 k^{2}-1536}}{32}<v-\frac{1}{v}<\frac{k^{2}}{3}$, the solitary wave solution $u_{1}(\xi)$ is orbital stable; if $b_{2}<0$, when $0<v-\frac{1}{v}<\frac{-7 k^{4}+\sqrt{241 k^{2}-1536}}{32}$, the solitary wave solution $u_{1}(\xi)$ is orbital instable.

The conclusion for $\bar{d}^{\prime \prime}(v)$ can be obtained after similar discussion.

Theorem 5 Suppose $p=1, b_{3}>0, k=\frac{\sqrt{2} b_{2}}{3 b_{3}}$.

(1) When $v-\frac{1}{v} \geq \frac{k^{2}}{3}$, whether $b_{2}>0$ or $b_{2}<0$, the solitary wave solution $u_{2}(\xi)$ is always orbital stable.

(2) When $v-\frac{1}{v}<\frac{k^{2}}{3}$, if $b_{2}<0$, when $\frac{-7 k^{4}+\sqrt{241 k^{2}-1536}}{32}<v-\frac{1}{v}<\frac{k^{2}}{3}$, the solitary wave solution $u_{2}(\xi)$ is orbital stable; if $b_{2}>0$, when $0<v-\frac{1}{v}<\frac{-7 k^{4}+\sqrt{241 k^{2}-1536}}{32}$, the solitary wave solution $u_{2}(\xi)$ is orbital instable.

\section{The Orbital Stability of the Solitary Wave Solution to Eq. (5) When $\boldsymbol{p}>\mathbf{2}$}

When $p>2$, we have $k=\frac{b_{2} \sqrt{p+1}}{\sqrt{b_{3}}(p+2)}$ and 


$$
u_{i}(\xi)=\left(\frac{A_{i} \operatorname{sech}^{2} \frac{\alpha}{2}\left(\xi+\xi_{0}\right)}{2+B_{i} \operatorname{sech}^{2} \frac{\alpha}{2}\left(\xi+\xi_{0}\right)}\right)^{1 / p}(\xi=x-v t) .
$$

Substituting (32) into (24), we can get

$$
d^{\prime}(v)=\frac{1}{2} \int_{R}\left(\left(1+\frac{1}{v^{2}}\right)\left(\frac{A_{i} \operatorname{sech}^{2} \frac{\alpha}{2} \xi}{2+B_{i} \operatorname{sech}^{2} \frac{\alpha}{2} \xi}\right)^{2 / p}+\frac{4 A_{i}^{\frac{2}{p}} \alpha^{2} \tanh ^{2} \frac{\alpha}{2} \xi \operatorname{sech}^{\frac{4}{p}} \frac{\alpha}{2} \xi}{p^{2}\left(2+B_{i} \operatorname{sech}^{2} \frac{\alpha}{2} \xi\right)^{\frac{2}{p}+2}}\right) d \xi
$$

Because of the complexity of the integral formula, we can not obtain the stable or instable velocity changing interval directly. So we discuss the sign of discriminant by using reasonable expansion and contraction here. At the same time, without losing generality, we only discuss the orbit stability of solitary wave solution (7). The orbit stability of solitary wave solution (10) can be discussed similarly.

\subsection{The Discriminant of Orbital Stability of the Solitary Wave Solution (7)}

First, solitary wave solution $u_{1}(\xi)$ can be rewritten as

$$
u_{1}(\xi)=\left(\frac{1}{d_{1}+d_{2} \cosh ^{2} \frac{d_{3}}{2}\left(\xi+\xi_{0}\right)}\right)^{\frac{1}{p}},(\xi=x-v t),
$$

where

$$
\begin{aligned}
& d_{1}=\frac{B_{1}}{A_{1}}=\frac{b_{2} \sqrt{p+1}-\sqrt{b_{2}^{2}(p+1)+b_{3}(p+2)^{2} m}}{\sqrt{p+1}(p+2) m} \\
& d_{2}=\frac{2}{A_{1}}=\frac{2 \sqrt{b_{2}^{2}(p+1)+b_{3}(p+2)^{2} m}}{\sqrt{p+1}(p+2) m}>0, \\
& \alpha=p \sqrt{\frac{m}{v}},
\end{aligned}
$$

and $m=v-\frac{1}{v}$. Let $M=d_{1}+d_{2} \cosh ^{2} \frac{d_{3}}{2}\left(\xi+\xi_{0}\right)$, since

$$
\begin{gathered}
u_{1 \xi}^{2}=\frac{m d_{2}^{2} \cosh ^{2} \frac{d_{3}}{2} \xi \sinh ^{2} \frac{d_{3}}{2} \xi}{v M^{\frac{2}{p}+2}}=\frac{m d_{2}^{2} \cosh ^{2} \frac{d_{3}}{2} \xi\left(\cosh ^{2} \frac{d_{3}}{2} \xi-1\right)}{v M^{\frac{2}{p}+2}} \\
=\frac{m\left(M^{2}-\left(2 d_{1}+d_{2}\right) M+d_{1}\left(d_{1}+d_{2}\right)\right)}{v M^{\frac{2}{p}+2}},
\end{gathered}
$$

and consider $\cosh \left(d_{3} x / 2\right)$ is a even function, so $d^{\prime}(v)$ can be rewritten as

$$
d^{\prime}(v)=\int_{0}^{+\infty}\left(\frac{2}{M^{\frac{2}{p}}}-\frac{m}{v} \frac{2 d_{1}+d_{2}}{M^{\frac{2}{p}+1}}+\frac{m}{v} \frac{d_{1}\left(d_{1}+d_{2}\right)}{M^{\frac{2}{p}+2}}\right) d \xi .
$$

For $n>0$, we have 


$$
\begin{aligned}
\int_{0}^{+\infty} \frac{\xi \cosh \frac{d_{3}}{2} \xi \sinh \frac{d_{3}}{2} \xi}{M^{n+1}} d \xi= & \frac{1}{n d_{2} d_{3}} \int_{0}^{+\infty} \frac{1}{M^{n}} d \xi \\
\int_{0}^{+\infty} \frac{\partial}{\partial v}\left(\frac{1}{M^{n}}\right) d \xi= & -\int_{0}^{+\infty} \frac{n M^{n-1}}{M^{2 n}} \frac{\partial M}{\partial v} d \xi \\
= & -\left(n \frac{d^{\prime}}{d_{2}}+\frac{d_{3}^{\prime}}{d_{3}}\right) \int_{0}^{+\infty} \frac{1}{M^{n}} d \xi \\
& -n\left(d^{\prime}{ }_{1}-\frac{d_{2}^{\prime}}{d_{2}} d_{1}\right) \int_{0}^{+\infty} \frac{1}{M^{n+1}} d \xi
\end{aligned}
$$

From (37), we know that $\int_{0}^{+\infty} \frac{\partial}{\partial v}\left(\frac{1}{M^{n}}\right) d \xi$ converges when $v$ satisfies $v^{2}>1$. Because for any real number $\alpha, \beta>0$, we have $\frac{p}{\beta} \sqrt{\beta^{2}-1}<d_{3}<\frac{p}{\gamma} \sqrt{\gamma^{2}-1}$, then when $v$ satisfies $1<\beta<v^{2}<\gamma, \cosh \left(\frac{p}{2 \beta} \sqrt{\beta^{2}-1} \xi\right)<\cosh \left(\frac{d_{3} \xi}{2}\right)$ on $(0,+\infty)$, and there exist a constant $K$ independent of $v$ such that

$$
\begin{aligned}
& 0<\frac{1}{d_{1}+d_{2} \cosh ^{2}\left(d_{3} \xi / 2\right)}<\frac{1}{d_{1}+d_{2} \cosh ^{2}\left(p \sqrt{\beta^{2}-1} \xi / 2 \beta\right)} \\
& =\frac{4 e^{\frac{p}{\beta} \sqrt{\beta^{2}-1 \xi}}}{d_{2} e^{\frac{p}{\beta} \sqrt{\beta^{2}-1} \xi}\left(1+2\left(\frac{2 d_{1}}{d_{2}}+1\right) e^{\frac{p}{\beta} \sqrt{\beta^{2}-1} \xi}+e^{\frac{2 p}{\beta} \sqrt{\beta^{2}-1} \xi}\right)}<\frac{4 K}{e^{\frac{p}{\beta} \sqrt{\beta^{2}-1 \xi}} .}
\end{aligned}
$$

So $\int_{0}^{+\infty} \frac{\partial}{\partial v}\left(\frac{1}{M^{n}}\right) d \xi$ inner closed uniform converges when $v>1$. Then according to (36) (37), we can get the discriminant of the solitary wave solution (7) to Eq. (5) as follows,

$$
\begin{aligned}
d^{\prime \prime}(v) & =\int_{0}^{+\infty} \frac{\partial}{\partial v}\left(\frac{2}{M^{\frac{2}{p}}}-\frac{m}{v} \frac{2 d_{1}+d_{2}}{M^{\frac{2}{p}+1}}+\frac{m}{v} \frac{d_{1}\left(d_{1}+d_{2}\right)}{M^{\frac{2}{p}+2}}\right) d \xi \\
& =\int_{0}^{+\infty} \sum_{i=0}^{3} \frac{\beta_{i}}{M^{\frac{2}{p}+i}} d \xi
\end{aligned}
$$

where

$$
\begin{gathered}
\beta_{0}=2 \frac{k^{2}\left(2 v^{2}+2-p\right)+m\left(v^{2}+1-p\right)}{p v^{2} m\left(k^{2}+m\right)}, \\
\beta_{1}=\frac{b_{2}\left[4 v-2 k^{2}\left(2 v^{2}+2+p\right)+m\left(p v^{2}-p-2\right)\right]}{(p+2) p v^{3} m\left(k^{2}+m\right)}, \\
\beta_{2}=-\frac{b_{3}}{p\left(k^{2}+m\right)(p+1) v^{3} m}\left(k^{2}\left(2(2+p) v^{2}+4+3 p\right)+m\left(v^{2}+1+p\right)\right),
\end{gathered}
$$




$$
\beta_{3}=-\frac{b_{2} b_{3}\left(v^{2}+1\right)}{p(p+2)\left(k^{2}+m\right) v^{3} m},
$$

and here $m=v-\frac{1}{v}, \quad k=\frac{b_{2} \sqrt{p+1}}{\sqrt{b_{3}}(p+2)}$. Then we only need to consider the sign of $d^{\prime \prime}(v)$.

Remark 3 If we take $b_{2}=1, b_{3}=0$ in (36), then $B_{i}=0, d_{1}=0$. Now we can get

$$
d^{\prime}(v)=\left(\frac{(p+2) m}{2}\right)^{\frac{2}{p}} \int_{0}^{+\infty}\left(2 \operatorname{sech}^{\frac{4}{p}} \frac{d_{3}}{2}\left(\xi+\xi_{0}\right)-\frac{m}{v} \operatorname{sech}^{\frac{4}{p}}+2 \frac{d_{3}}{2}\left(\xi+\xi_{0}\right)\right) d \xi
$$

According to the appropriate transformation and Wallis formula, we can deduce the conclusion which is consistent with Theorem 6.1 in reference [17].

\subsection{The Orbital Stability of Solitary Wave Solution (7) When $b_{2}>0$}

Now we start to judge the sign of determinant of solitary wave solution (7) to Eq. (5). When $b_{2}>0$, it's easy to get that $\beta_{2}<0, \beta_{3}<0$.

When $b_{2}>0, b_{3}>0$, let $\beta_{1}=\beta_{11}+\beta_{12}$ in (39b), where

$$
\begin{gathered}
\beta_{11}=\frac{b_{2}\left[4 v+m\left(p v^{2}-p-2\right)\right]}{p v^{3} m(p+2)\left(k^{2}+m\right)}>0, \\
\beta_{12}=-\frac{2 b_{2} k^{2}\left(2 v^{2}+2+p\right)}{p v^{3} m(p+2)\left(k^{2}+m\right)}<0 .
\end{gathered}
$$

Since $0<\frac{d_{1}+d_{2}}{d_{1}+d_{2} \cosh ^{2}\left(d_{3} \xi / 2\right)}<1$, we have

$$
\int_{0}^{+\infty}\left(\frac{1}{d_{1}+d_{2} \cosh ^{2}\left(d_{3} \xi / 2\right)}\right)^{\frac{2}{p}+1} d \xi<\frac{1}{d_{1}+d_{2}} \int_{0}^{+\infty}\left(\frac{1}{d_{1}+d_{2} \cosh ^{2}\left(d_{3} \xi / 2\right)}\right)^{\frac{2}{p}} d \xi
$$

By substituting (41) (42) into (38), we have

$$
d^{\prime \prime}(v) \leq \int_{0}^{+\infty} \frac{1}{M^{\frac{2}{p}}}\left(\beta_{0}+\frac{\beta_{11}}{d_{1}+d_{2}}\right) d \xi .
$$

Now set $G_{1}=\beta_{0}+\frac{\beta_{11}}{d_{1}+d_{2}}$, and substitute (39a) (41a) into $G_{1}$, we can obtain 


$$
\begin{aligned}
G_{1}= & \frac{2}{p v^{3}\left(v^{2}-1\right)\left(k^{2}+m\right)}\left[k^{2}\left(2 v^{4}+(2-p) v^{2}\right)+m\left(v^{4}+(1-p) v^{2}\right)\right. \\
& \left.+\frac{k m}{k+\sqrt{k^{2}+m}}\left(p\left(v^{2}-1\right)+2\left(v^{2}-1\right)+4\right)\right] \\
< & \frac{2}{p v^{3}\left(v^{2}-1\right)\left(k^{2}+m\right)}\left[k^{2}\left(2 v^{4}+(2-p) v^{2}\right)\right. \\
& \left.+m\left(\left(\frac{p}{2}+1\right) v^{4}-2(p-1) v^{2}+\frac{p}{2}+1\right)\right] \\
= & \frac{2}{p v\left(v^{2}-1\right)\left(k^{2}+m\right)}\left[k^{2}\left(2 v^{2}+2-p\right)\right. \\
& \left.+m\left(\left(\frac{p}{2}+1\right)\left(v^{2}+\frac{1}{v^{2}}\right)-2(p-1)\right)\right] .
\end{aligned}
$$

From (44), we can get $G_{1}<0$ when

$$
\left\{\begin{array}{l}
2 v^{2}+2-p<0 \\
\left(\frac{p}{2}+1\right)\left(v^{2}+\frac{1}{v^{2}}-2\right)-p+4<0
\end{array}\right.
$$

are established. Then it can be deduced that when $p>4$, if $0<v-\frac{1}{v}<\min \left\{\frac{p-4}{\sqrt{2 p-4}}, \frac{\sqrt{2(p-4)}}{\sqrt{p+2}}\right\}$, we have $G_{1}<0$, namely $d^{\prime \prime}(v)<0$.

Next we consider when the discriminant of (5) is positive. From the above analysis, we can know that $\beta_{11}>0$, so from (41a), we have

$$
d^{\prime \prime}(v) \geq \int_{0}^{+\infty} \frac{1}{M^{\frac{2}{p}}}\left(\beta_{0}+\frac{\beta_{12}}{d_{1}+d_{2}}+\frac{\beta_{2}}{\left(d_{1}+d_{2}\right)^{2}}+\frac{\beta_{3}}{\left(d_{1}+d_{2}\right)^{3}}\right) d \xi,
$$

Let $G_{2}=\beta_{0}+\frac{\beta_{12}}{d_{1}+d_{2}}+\frac{\beta_{2}}{\left(d_{1}+d_{2}\right)^{2}}+\frac{\beta_{3}}{\left(d_{1}+d_{2}\right)^{3}}$, and substitute (38) (41b) into $G_{2}$, we can get

$$
\begin{aligned}
M_{0} G_{2}= & 2 k^{3}\left(k+\sqrt{k^{2}+m}\right)^{2}\left((2-2 p) v^{2}+p+2\right) \\
& +k^{2} m\left(k+\sqrt{k^{2}+m}\right)\left((12-2 p) v^{4}+(16-13 p) v^{2}+3 p+4\right) \\
& +k m^{2}\left((p+14) v^{4}+(12-7 p) v^{2}-2 p-2\right) \\
& +m^{2} \sqrt{k^{2}+m}\left(5 v^{4}+(6-5 p) v^{2}+p+1\right)
\end{aligned}
$$

where $\quad M_{0}=p\left(k^{2}+m\right) v^{2}\left(v^{2}-1\right)\left(k+\sqrt{k^{2}+m}\right)^{3}>0 . \quad$ By the relations $k^{2}<\sqrt{\left(k^{2}+m\right) k^{2}}<k^{2}+\frac{m}{2}$ and $m<\sqrt{m^{2}+4}<m+2$, (47) can be rewritten into 


$$
\begin{aligned}
M_{0} G_{2}= & k^{2}\left(k+\sqrt{k^{2}+m}\right) v^{4}\left(k\left(k+\sqrt{k^{2}+m}\right)\left((p+2) m^{2}-(p+2) m \sqrt{m^{2}+4}+8-2 p\right)\right. \\
& \left.+m\left(\left(8+\frac{p}{2}\right) m^{2}+\left(4-\frac{5 p}{2}\right) m \sqrt{m^{2}+4}+24-12 p\right)\right) \\
& +k m^{2}\left((p+14) v^{4}+(12-7 p) v^{2}-2 p-2\right) \\
& +m^{2} \sqrt{k^{2}+m}\left(5 v^{4}+(6-5 p) v^{2}+p+1\right) \\
> & k^{2}\left(k+\sqrt{k^{2}+m}\right) v^{4}\left(7 m^{3}+\left(2-\frac{7}{2} p\right) m^{2}\right. \\
& \left.+\left(8-6 p-4(p+2) k^{2}\right) m+4(4-p) k^{2}\right) \\
& +k m^{2} v^{2}\left((p+14) m^{2}+24-8 p\right)+m^{2} v^{2} \sqrt{k^{2}+m}\left(5 m^{2}+12-4 p\right) .
\end{aligned}
$$

Now, we let

$$
\begin{aligned}
G_{2}^{*}=k^{2} & \left(k+\sqrt{k^{2}+m}\right) v^{4}\left(7 m^{3}+\left(2-\frac{7}{2} p\right) m^{2}\right. \\
& \left.+\left(8-6 p-4(p+2) k^{2}\right) m+4(4-p) k^{2}\right) \\
& +k m^{2} v^{2}\left((p+14) m^{2}+24-8 p\right)+m^{2} v^{2} \sqrt{k^{2}+m}\left(5 m^{2}+12-4 p\right) .
\end{aligned}
$$

and consider when $G_{2}^{*}>0$ is established, that is to consider when to satisfy the following three inequalities,

$$
\left\{\begin{array}{l}
g_{1}(m)>0 \\
(p+14) m^{2}+24-8 p>0 \\
5 m^{2}+12-4 p>0
\end{array}\right.
$$

where $g_{1}(m)=7 m^{3}+\left(2-\frac{7}{2} p\right) m^{2}+\left(8-6 p-4(p+2) k^{2}\right) m+4(4-p) k^{2}$.

Set $\alpha_{i}(i=1,2,3)$ as three zeros of $g_{1}(m)=0$. Without loss of generality, here $\alpha_{1}<\alpha_{2}<\alpha_{3}$. According to Shengjin formula, we can get the conditions of existence of the three zeros $\alpha_{i}(i=1,2,3)$ as follows, $\Delta_{g 1}=B^{2}-4 A C<0$, where $A=-164+112 p+\frac{7}{4} p^{2}+84(p+2) k^{2}, B=21 p^{2}-40 p+16+2\left(7 p^{2}+136 p-512\right)$, $C=16(p+2)^{2} k^{4}+(8-4 p)^{2}+2 k^{2}\left(3 p^{2}+112 p-112\right)$. Assume $k, \quad p \quad$ satisfy $\Delta_{g 1}=B^{2}-4 A C<0$, so $g_{1}(m)$ can be rewritten into

$$
g_{1}(m)=7\left(m-\alpha_{1}\right)\left(m-\alpha_{2}\right)\left(m-\alpha_{3}\right) .
$$

Then by deriving $g_{1}(m)$ with respect to $m$, we can get

$$
\frac{\partial g_{1}(m)}{\partial m}=21 m^{2}+(4-7 p) m+8-6 p-4(p+2) k^{2},
$$

with solutions,

$$
m_{ \pm}=\frac{7 p-4 \pm \sqrt{(4-7 p)^{2}+84\left(4(p+2) k^{2}+6 p-8\right)}}{42}
$$


where $m_{-}<0<m_{+}$. When $m<m_{-}$or $m>m_{+}, g_{1}(m)$ is monotonically increasing; when $m_{-}<m<m_{+}, g_{1}(m)$ is monotonically decreasing. So we can deduce the approximate location of three points,

$$
\begin{gathered}
\alpha_{1}<\frac{7 p-4-\sqrt{(4-7 p)^{2}+84\left(4(p+2) k^{2}+6 p-8\right)}}{42}<0, \\
\quad \frac{7 p-4-\sqrt{(4-7 p)^{2}+84\left(4(p+2) k^{2}+6 p-8\right)}}{42}<\alpha_{2} \\
<\frac{7 p-4+\sqrt{(4-7 p)^{2}+84\left(4(p+2) k^{2}+6 p-8\right)}}{42}, \\
\alpha_{3}>\frac{7 p-4+\sqrt{(4-7 p)^{2}+84\left(4(p+2) k^{2}+6 p-8\right)}}{42},
\end{gathered}
$$

and know when $m \in\left(-\infty, \alpha_{1}\right) \cup\left(\alpha_{2}, \alpha_{3}\right), g_{1}(m)<0$; when $m \in\left(\alpha_{1}, \alpha_{2}\right) \cup\left(\alpha_{3},+\infty\right)$, $g_{1}(m)>0$. So when $2<p \leq 4$, we have

$$
\begin{aligned}
& \lim _{t \rightarrow 0^{+}} g_{1}(m)=4(4-p) k>0, \\
& g_{1}(1)=17-\frac{19}{2} p-8 k p+8 k<0 .
\end{aligned}
$$

So there exist a zero point $\alpha_{2}$ in the interval $(0,1)$, for any $m \in\left(0, \alpha_{2}\right), g_{1}(m)>0$. According to the properties of univariate cubic equation, we can get that when $m>\alpha_{3}, g_{1}(m)>0$.

Then consider the last two inequalities in (49), we can get when $2<p<3$,

$$
\left\{\begin{array}{l}
(p+14) m^{2}+24-8 p>0 \\
5 m^{2}+12-4 p>0
\end{array}\right.
$$

are always established. And when $p \geq 3$, we can get

$$
\left\{\begin{array}{l}
m>\sqrt{\frac{8 p-24}{p+14}}, \\
m>\sqrt{\frac{4 p-12}{5}} .
\end{array}\right.
$$

from (50). Since

$$
\sqrt{\frac{8 p-24}{p+14}}=\sqrt{\frac{2(4 p-12)}{p+14}}<\sqrt{\frac{4 p-12}{5}}
$$


when $2<p \leq 4$, we can obtain that when $2<p<3$ and $0<m<\alpha_{2}$, or when $3 \leq p<4$ and $\sqrt{\frac{4 p-12}{5}}<m<\alpha_{2}$, or when $p \geq 4$ and $m \geq \max \left\{\alpha_{3}, \sqrt{\frac{4 p-12}{5}}\right\}$, $G_{2}>0$.

According to the above analysis, we can get the following theorem.

Theorem 6 Suppose $b_{2}>0, b_{3}>0, p>2$, and $k$, p satisfy $\Delta_{g 1}=B^{2}-4 A C<0$.

(1) If $p>4$ and $v$ satisfies $0<v-\frac{1}{v}<\min \left\{\frac{p-4}{\sqrt{2 p-4}}, \frac{\sqrt{2(p-4)}}{\sqrt{p+2}}\right\}$, the solitary wave solution (7) of equation (5) is orbital instable.

(2) If $2<p<3$, when $v$ satisfies $0<v-\frac{1}{v}<\alpha_{2}$ or when $3 \leq p<4$ and $v$ satisfies $\sqrt{\frac{4 p-12}{5}}<v-\frac{1}{v}<\alpha_{2}$, the solution (7) of (5) is orbital stable.

(3) If $p \geq 4$, when $v-\frac{1}{v}>\max \left\{\alpha_{3}, \sqrt{\frac{4 p-12}{5}}\right\}$, the solitary wave solution (7) of (5) is orbital stable.

\subsection{The Orbital Stability of Solitary Wave Solution (7) When $b_{2}<0$}

Obviously, when $b_{2}<0$, we have $\beta_{2}<0$ and $\beta_{11}<0$ from (39c) (41a). So in order to make $d^{\prime \prime}(v)<0$, we only need to consider when

$$
d^{\prime \prime}(v) \leq \int_{0}^{+\infty} \frac{1}{M^{\frac{2}{p}}}\left(\beta_{0}+\frac{\beta_{12}}{d_{1}+d_{2}}+\frac{\beta_{3}}{\left(d_{1}+d_{2}\right)^{3}}\right) d \xi
$$

is established. Set $G_{3}=\beta_{0}+\frac{\beta_{12}}{d_{1}+d_{2}}+\frac{\beta_{3}}{\left(d_{1}+d_{2}\right)^{3}}$, we can get

$$
\begin{aligned}
G_{3}= & \frac{2 k^{3}\left((2-2 p) v^{2}+2+p\right)+2 k^{2} \sqrt{k^{2}+m}\left(2 v^{4}+(2-p) v^{2}\right)}{p\left(k^{2}+m\right) v^{3}\left(v^{2}-1\right)\left(k+\sqrt{k^{2}+m}\right)} \\
& +\frac{2\left(4 k^{3} m+4 k^{2} m \sqrt{k^{2}+m}+m^{2} \sqrt{k^{2}+m}\right)\left(v^{4}+(1-p) v^{2}\right)+k m^{2}\left((5-p) v^{4}+6(1-p) v^{2}+p+1\right)}{p\left(k^{2}+m\right) v^{3}\left(v^{2}-1\right)\left(k+\sqrt{k^{2}+m}\right)} .
\end{aligned}
$$

By the relation $m<\sqrt{m^{2}+4}<m+2, G_{3}$ can be expressed as follows,

$$
\begin{aligned}
G_{3}< & \frac{k^{3} v\left((4-p) \sqrt{m^{2}+4}-3 p m\right)+k^{2} v^{3} \sqrt{k^{2}+m}\left((4-p) \sqrt{m^{2}+4}+p m\right)}{p\left(k^{2}+m\right) v^{3}\left(v^{2}-1\right)\left(k+\sqrt{k^{2}+m}\right)} \\
& +\frac{2\left(4 k^{3} m+4 k^{2} m \sqrt{k^{2}+m}+m^{2} \sqrt{k^{2}+m}\right) v^{3}\left(\left(1-\frac{p}{2}\right) \sqrt{m^{2}+4}+\frac{p}{2} m\right)+k m^{2} v^{2}\left((5-p) m^{2}+2(2-p) m+6(2-p)\right)}{p\left(k^{2}+m\right) v^{3}\left(v^{2}-1\right)\left(k+\sqrt{k^{2}+m}\right)^{3}} .
\end{aligned}
$$

Set $g_{2}(m)=(5-p) m^{2}+2(2-p) m+6(2-p), \quad$ since $\quad b_{2}<0, \quad k=\frac{b_{2} \sqrt{p+1}}{\sqrt{b_{3}}(p+2)}<0$, $G_{3}<0$ is equivalent to 


$$
\left\{\begin{array}{l}
(4-p) \sqrt{m^{2}+4}-3 p m>0, \\
(4-p) \sqrt{m^{2}+4}+p m<0, \\
g_{2}(m)>0
\end{array}\right.
$$

where $2<p<4$. Since $\frac{p-2-\sqrt{(p-2)(28-5 p)}}{5-p}<0$, we can obtain

$$
\left\{\begin{array}{l}
m^{2}<\frac{(4-p)^{2}}{2(p+2)(5 p-2)}, \\
m^{2}<\frac{(p-4)^{2}}{2 p-4}, \\
m>\frac{p-2+\sqrt{(p-2)(28-5 p)}}{5-p}
\end{array}\right.
$$

from (53), namely $\frac{p-2+\sqrt{(p-2)(28-5 p)}}{5-p}<v-\frac{1}{v}<\frac{4-p}{\sqrt{2(p+2)(5 p-2)}}$.

When $b_{2}<0, p>2$, we have $\beta_{3}>0, \beta_{12}>0$. From (48), we have

$$
d^{\prime \prime}(v) \geq \int_{0}^{+\infty} \frac{1}{M^{\frac{2}{p}}}\left(\beta_{0}+\frac{\beta_{11}}{d_{1}+d_{2}}+\frac{\beta_{2}}{\left(d_{1}+d_{2}\right)^{2}}\right) d \xi .
$$

Set $G_{4}=\beta_{0}+\frac{\beta_{11}}{d_{1}+d_{2}}+\frac{\beta_{2}}{\left(d_{1}+d_{2}\right)^{2}}$, we have

$$
\begin{aligned}
\frac{M_{0} G_{4}}{k+\sqrt{k^{2}+m}}= & 2 k^{3}\left(k+\sqrt{k^{2}+m}\right) v^{2}\left(2 v^{2}+2-p\right) \\
& +k m \sqrt{k^{2}+m}\left((p+4) v^{4}+6(1-p) v^{2}+p+2\right) \\
& +k^{2} m\left((4-p) v^{4}+(10-9 p) v^{2}+4 p+6\right) \\
& +m^{2}\left(v^{4}+(2-3 p) v^{2}+1+p\right)
\end{aligned}
$$

and from relation $m<\sqrt{m^{2}+4}<m+2$, the above equation can be rewritten into

$$
\begin{aligned}
\frac{M_{0} G_{4}}{k+} & \sqrt{k+m^{2}} \\
= & k^{3}\left(k+\sqrt{k+m^{2}}\right) v^{3}\left((4-p) \sqrt{m^{2}+4}+p m\right) \\
& +k m \sqrt{k+m^{2}}\left((p+3) m^{2}+m \sqrt{m^{2}+4}+12-4 p\right) \\
& +\frac{k^{2} m v^{2}}{2}\left((3 p+10) m^{2}-(5 p+2) m \sqrt{m^{2}+4}+20-6 p\right) \\
& +m^{2} v^{2}\left(\left(\frac{p}{2}+1\right) m^{2}-\frac{p m \sqrt{m^{2}+4}}{2}+4-2 p\right) \\
> & 2 k^{3} v^{3}\left(k+\sqrt{k+m^{2}}\right)\left(\left(2-\frac{p}{2}\right) \sqrt{m^{2}+4}+\frac{p}{2} m\right)+m v^{2} g_{3}(m) \\
& +k m \sqrt{k+m^{2}} v^{2}\left((p+4) m^{2}+2 m+4(3-p)\right)
\end{aligned}
$$


where

$$
g_{3}(m)=\frac{p+2}{2} m^{3}+\left[(4-p) k^{2}-\frac{p}{2}\right] m^{2}+\left(4-3 p-(5 p+2) k^{2}\right) m+(20-6 p) k^{2} .
$$

Then we only need to consider when

$$
\left\{\begin{array}{l}
(4-p) \sqrt{m^{2}+4}+p m<0 \\
(p+4) m^{2}+2 m+4(3-p)<0 \\
g_{3}(m)>0
\end{array}\right.
$$

are established. For the first two inequalities, we have

$$
\left\{\begin{array}{l}
m<\frac{p-4}{\sqrt{2 p-4}}, \\
0<m<\frac{-1+\sqrt{4 p^{2}+4 p-47}}{p+4} .
\end{array}\right.
$$

Then set three zeroes of $g_{3}(m)$ as $\bar{\alpha}_{i}(i=1,2,3)\left(\bar{\alpha}_{1}<\bar{\alpha}_{2}<\bar{\alpha}_{3}\right)$, and $g_{3}(m)$ can be rewritten into

$$
g_{3}(m)=\frac{p+2}{2}\left(m-\bar{\alpha}_{1}\right)\left(m-\bar{\alpha}_{2}\right)\left(m-\bar{\alpha}_{3}\right) .
$$

Similar to the discussion of $g_{1}(m)$, we consider the positions of $\bar{\alpha}_{i}(i=1,2,3)$. By deriving the $g_{3}(m)$ with respect to $m$, we have

$$
\frac{\partial g_{3}(m)}{\partial m}=\frac{3(p+2)}{2} m^{2}+\left[(8-2 p) k^{2}-p\right] m+4-3 p-(5 p+2) k^{2},
$$

and

$$
\begin{aligned}
\Delta & =\left[(8-2 p) k^{2}-p\right]^{2}-6(p+2)\left(4-3 p-(5 p+2) k^{2}\right)>\left[(8-2 p) k^{2}-p\right]^{2}, \\
m_{+} & =\frac{(2 p-8) k^{2}+p+\sqrt{\Delta}}{3(p+2)}>0, m_{-}=\frac{(2 p-8) k^{2}+p-\sqrt{\Delta}}{3(p+2)}<0 .
\end{aligned}
$$

So we can get the positions of three zeroes as follows,

$$
\bar{\alpha}_{1}<m_{-}<\bar{\alpha}_{2}<m_{+}<\bar{\alpha}_{3} .
$$

Since

$$
\begin{aligned}
& \lim _{m \rightarrow 0^{+}} g_{3}(m)=(20-6 p) k^{2} \geq 0, \\
& \lim _{m \rightarrow 1} g_{3}(m)=g_{3}(1)=5-3 p+(22-12 p) k^{2}<0,
\end{aligned}
$$

we have $\bar{\alpha}_{2} \in(0,1)$, and when $m \in\left(0, \bar{\alpha}_{2}\right), g_{3}(m)>0$. Besides, when $m>\bar{\alpha}_{3}$, $g_{3}(m)>0$.

Combining the above analysis, we can get the following theorem. 
Theorem 7 Set $p>2, b_{2}<0$. (1) In the case of $2<p<4$, the solitary wave solution (7) is orbital instable when $\frac{p-2+\sqrt{(p-2)(28-5 p)}}{5-p}<v-\frac{1}{v}<\frac{4-p}{\sqrt{2(p+2)(5 p-2)}}$.

(2) In the case of $3<p \leq \frac{10}{3}$, there exists $\bar{\alpha}_{2}\left(0<\bar{\alpha}_{2}<1\right)$ such that for any $\frac{p-4}{\sqrt{2 p-4}}<v-\frac{1}{v}<\min \left\{\bar{\alpha}_{2}, \frac{-1+\sqrt{4 p^{2}+4 p-47}}{p+4}\right\}$, the solitary wave solution (7) is orbital stable.

(3) In the case of $p>\frac{10}{3}$, there exists $\bar{\alpha}_{3}\left(\bar{\alpha}_{3}<\frac{-1+\sqrt{4 p^{2}+4 p-47}}{p+4}\right)$ such that for any $\bar{\alpha}_{3}<v-\frac{1}{v}<\frac{-1+\sqrt{4 p^{2}+4 p-47}}{p+4}$, the solitary wave solution $(7)$ is orbital stable.

\section{The Influence of Two Nonlinear Terms on the Stability of the Solitary Wave Solution}

In this section, we consider the influence of the two nonlinear terms on the orbital stability of the solitary wave solution to the gsrlw Eq. (5).

From the conclusions in the Sects. 3, 4, it can be found that the stable and instable wave velocity intervals are mainly determined by two factors, one is the coefficient of the nonlinear term, the other is the order of the nonlinear term. Here we discuss the influence of the coefficient of the nonlinear term first.

Without loss of generality, we take the solitary wave solution (7) as an example. According to the Theorems 2 and 4, we can get that the stable and instable wave velocity intervals, which are shown in the following tables.

It can be found that when there are two nonlinear terms, the stable and unstable intervals of the solitary wave solutions are affected by the ratio $b_{2} / \sqrt{b_{3}}$ of the coefficients $b_{2}, b_{3}$ of the nonlinear terms, but not by the coefficient of a single nonlinear term. In other words, if the ratio $b_{2} / \sqrt{b_{3}}$ of the two nonlinear terms is a constant and whether the coefficients of the two nonlinear terms are increasing or decreasing proportionally, the orbital stability and instability of the solitary wave solutions with two low order nonlinear terms will not be affected.

Besides, from Table 1 , we can find that when the ratio $\left|b_{2} / \sqrt{b_{3}}\right|$ is larger, the orbital stable interval will become smaller. Correspondingly, the orbital instable interval will become larger.

Table 1 The stable and instable wave velocity intervals of solution (7) to Eq. (5) when $p=1, b_{3}>0$, $k=\frac{\sqrt{2} b_{2}}{3 b_{3}}$

\begin{tabular}{lll}
\hline Coefficients & Stable interval & Instable interval \\
\hline$b_{2}>0$ & $\frac{-7 k^{4}+\sqrt{241 k^{2}-1536}}{32}<v-\frac{1}{v}<+\infty$ & Unknown \\
$b_{2}<0$ & $v-\frac{1}{v} \geq \frac{k^{2}}{3}$ & $0<v-\frac{1}{v}<\frac{-7 k^{4}+\sqrt{241 k^{2}-1536}}{32}$ \\
\hline
\end{tabular}


Table 2 The stable and instable wave velocity intervals of solution (7) to Eq. (5) when $b_{3}>0, p=2$, $k=\frac{\sqrt{3} b_{2}}{4 \sqrt{b_{3}}}$

\begin{tabular}{|c|c|c|}
\hline Coefficients & Stable interval & Instable interval \\
\hline$b_{2}>0$ & $\begin{array}{l}\frac{9}{v}-v<k^{2}<v-\frac{1}{v}(\sqrt{5}<v<3) \text { or } \\
\frac{\left(v^{2}-1\right)^{2}\left(v-\frac{9}{v}\right)}{v^{4}-6 v^{2}+13}<k^{2}<v-\frac{1}{v}(v>3)\end{array}$ & $\begin{array}{l}\max \left\{v-\frac{1}{v}, \frac{9}{v}-v\right\}<k^{2}<\frac{\left(v^{2}-1\right)^{2}+4 v^{2}+4}{v\left(v^{2}-1\right)} \\
(v<3) \\
v-\frac{1}{v}<k^{2}<\frac{\left(v^{2}-1\right)^{2}+4 v^{2}+4}{v\left(v^{2}-1\right)}(v>3)\end{array}$ \\
\hline$b_{2}<0$ & Unknown & $k^{2}>\frac{\left(v^{2}-1\right)^{2}+4 v^{2}+4}{v\left(v^{2}-1\right)}$ or $k^{2}<v-\frac{1}{v}$ \\
\hline
\end{tabular}

For Table 2, since the conclusions are complex, we give its corresponding figure to help us analyze the influence from the ratio of coefficients of nonlinear terms on the stability interval.

As it is shown in Fig. 1, when the ratio $\left|b_{2} / \sqrt{b_{3}}\right|$ is between the line $L_{2}$ and $L_{3}$, the solution (7) is orbital stable. When $1<v<3$ and the ratio $\left|b_{2} / \sqrt{b_{3}}\right|$ is below the line $l_{1}$, and above the maximum of line $L_{2}$ and $L_{3}$, or $v>3$ and the ratio is between the line $L_{1}$ and $L_{2}$, the solution is orbital instable.

From Fig. 1, we can find that if when the ratio $\left|b_{2} / \sqrt{b_{3}}\right|$ becomes larger, the stable wave interval will become larger first, and the instable wave velocity interval does not exist; then with the increasing of the ratio $\left|b_{2} / \sqrt{b_{3}}\right|$, the instable wave velocity interval appears and becomes larger, while the stable wave velocity becomes smaller; as the ratio $\left|b_{2} / \sqrt{b_{3}}\right|$ exceeds a certain value, the stable wave interval will disappear, and the instable wave velocity will start to become smaller. Obviously, the changing of the stable and instable wave velocity intervals for the case of $p=2$ is more complex than the case of $p=1$.

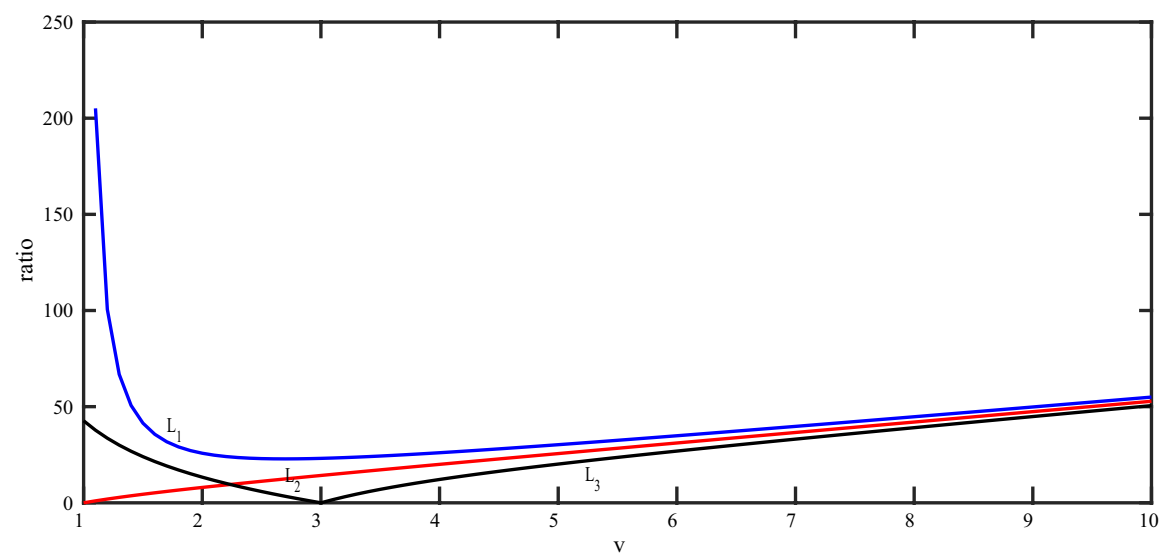

Fig. 1 Diagram of the stable and instable wave velocity intervals when $b_{3}>0, p=2$ 
Then we discuss the influence of the order of nonlinear term. According to Theorem 6, when $p>4$, the instable wave velocity interval of solitary wave solution (7) to Eq. (5) is $0<v-\frac{1}{v}<\min \left\{\frac{p-4}{\sqrt{2 p-4}}, \frac{\sqrt{2(p-4)}}{\sqrt{p+2}}\right\}$. It can be found that when the order $p(p>4)$ is increasing, the instable wave velocity interval of solution will become larger. Correspondingly, its stable wave velocity interval will become smaller.

Furthermore, it can be seen that the influence of the coefficients of the nonlinear terms on the orbital stability of solitary wave solution (7) is not revealed in the Theorem 6, which may caused by the over scaling of the discriminant $d^{\prime \prime}(v)$. In other words, after the scaling of the discriminant $d^{\prime \prime}(v)$, the order of the nonlinear terms still influence the orbital stable wave velocity intervals of the solitary wave solution (7) to Eq. (5), while the influence of the coefficients of the nonlinear terms cannot be shown. So we can guess that when the gsrlw equation has two high order nonlinear terms, the influence of the order of nonlinear terms on the orbital stability of solitary wave solution (7) is stronger than the influence of the coefficient of nonlinear terms.

Acknowledgements The authors will be grateful for comments from the referees and from the editor.

Author Contributions $\mathrm{WZ}$ and $\mathrm{XL}$ formal analysis and writing (original draft) the manuscript.

Funding This research is supported by National Nature Science Foundation of China (no. 11471215).

Data availability The authors confirm that the data supporting the findings of this study are available within the article and its supplementary materials.

\section{Declarations}

Conflict of interest No potential conflict of interest was reported by the authors.

Open Access This article is licensed under a Creative Commons Attribution 4.0 International License, which permits use, sharing, adaptation, distribution and reproduction in any medium or format, as long as you give appropriate credit to the original author(s) and the source, provide a link to the Creative Commons licence, and indicate if changes were made. The images or other third party material in this article are included in the article's Creative Commons licence, unless indicated otherwise in a credit line to the material. If material is not included in the article's Creative Commons licence and your intended use is not permitted by statutory regulation or exceeds the permitted use, you will need to obtain permission directly from the copyright holder. To view a copy of this licence, visit http://creativecommons.org/licen ses/by/4.0/.

\section{References}

1. Seyler, C.F., Fenstermmacher, D.C.: A symmetric regularized long wave equation. Phys. Fluids 27(1), 4-7 (1984)

2. Bogolubsky, I.L.: Cascade evolution of spherically symmetric pulsons in multivacuum field theory models. Phys. Lett. A 61(4), 205-206 (1977)

3. Makhankov, V.G.: Dynamics of classical solitons (in non-integrable systems). Phys. Rep. 35(1), $1-128$ (1978) 
4. Clarkson, P.A.: New similarity reductions and Painleve analysis for the symmetric regularized long wave and modified Benjamin-Bona-Mahoney equations. J. Phys. A Math. Gen. 22(18), 3821-3848 (1989)

5. Dianchen, L., Seadawy, A.R., Asghar, A.: Applications of exact traveling wave solutions of modified Liouville and the symmetric regularized long wave equations via two new techniques. Results Phys. 9, 1403-1410 (2018)

6. Khater, M., Abdelrahman, M.: Traveling solitary wave solutions for the symmetric regularized longwave equation. Adv. Math. 11(8), 5520-5528 (2015)

7. Manafian, J., Zamanpour, I.: Exact travelling wave solutions of the symmetric regularized long wave (SRLW) using analytical methods. Stat. Optim. Inf. Comput. 2014(2), 47-55 (2014)

8. Zhao, X.: An exponential wave integrator pseudospectral method for the symmetric regularizedlong-wave equation. J. Comput. Math. 34(1), 49-69 (2016)

9. Brango, C.B.: The symmetric regularized-long-wave equation: well-posedness and nonlinear stability. Phys. D 241(2), 125-133 (2012)

10. Pava, J.A., Brango, C.B.: Instability of periodic traveling waves for the symmetric regularized long wave equation. Nagoya Math. J. 219, 235-268 (2015)

11. Yadong, S.: Exact solutions of two kinds of nonlinear wave equations. J. Lanzhou Univ. 35(1), 11-17 (1999)

12. Demiray, H.: A note on the amplitude modulation of symmetric regularized long-wave equation with quartic nonlinearity. J. Eng. Math. 77(1), 181-186 (2012)

13. Montes, A.M., Cordoba, R.: Travelling waves for a generalized symmetric regularized-long-wave model. Turkish J. Electric. Eng. Comput. Sci. 22(4), 825-839 (2015)

14. Li, S., Wu, X.: $L^{\infty}$ error bound of conservative compact difference scheme for the generalized symmetric regularized long-wave (GSRLW) equations. Comput. Appl. Math. 37(2), 1-21 (2017)

15. Weiguo, Z.: Explicit exact solitary wave solutions for generalized symmetric regularized long-wave equations with high-order nonlinear terms. Chin. Phys. 12(2), 144-148 (2003)

16. Zhang, W., Chen, X., Li, Z., et al.: Orbital stability of solitary waves for generalized symmetric regularized-long-wave equations with two nonlinear terms. J. Appl. Math. 2014(1), 1-16 (2014)

17. Lin, Chen: Stability and instability of solitary waves for generalized symmetric regularized-longwave equations. Phys. D 118, 53-68 (1988)

18. Grillakis, M., Shatah, J., Strauss, W.: Stability theory of solitary waves in the presence of symmetry I. J. Funct. Anal. 74, 160-197 (1987)

19. Grillakis, M., Shatah, J., Strauss, W.: Stability theory of solitary waves in the presence of symmetry II. J. Funct. Anal. 94, 308-348 (1990)

20. Pazy, A.: Semigroups of Linear Operators and Applications to Partial Differential Equations. Springer, New York (1983) 